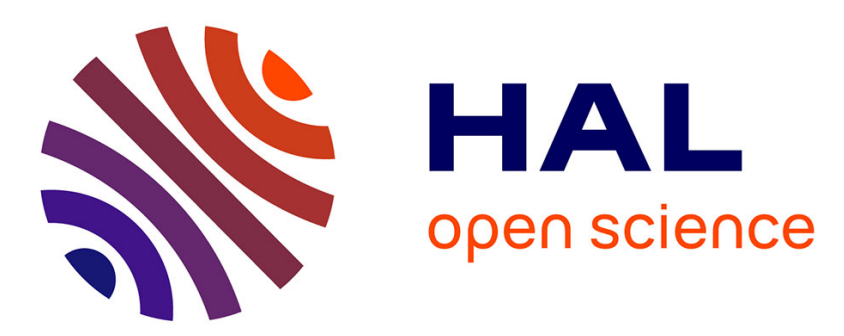

\title{
Confinement d'ions dans une cage cylindrique à champ quadrupolaire HF. Application à la spectrométrie de masse - II. Partie expérimentale
}

\author{
A. Galiouna Nassiopoulos, P.A. Möller, A. Septier
}

\section{- To cite this version:}

A. Galiouna Nassiopoulos, P.A. Möller, A. Septier. Confinement d'ions dans une cage cylindrique à champ quadrupolaire HF. Application à la spectrométrie de masse - II. Partie expérimentale. Revue de Physique Appliquée, 1980, 15 (10), pp.1543-1552. 10.1051/rphysap:0198000150100154300 jpa00244879

\section{HAL Id: jpa-00244879 https://hal.science/jpa-00244879}

Submitted on 1 Jan 1980

HAL is a multi-disciplinary open access archive for the deposit and dissemination of scientific research documents, whether they are published or not. The documents may come from teaching and research institutions in France or abroad, or from public or private research centers.
L'archive ouverte pluridisciplinaire HAL, est destinée au dépôt et à la diffusion de documents scientifiques de niveau recherche, publiés ou non, émanant des établissements d'enseignement et de recherche français ou étrangers, des laboratoires publics ou privés. 


\title{
Confinement d'ions dans une cage cylindrique à champ quadrupolaire HF. Application à la spectrométrie de masse
}

\section{Partie expérimentale}

\author{
A. Galiouna Nassiopoulos, P. A. Möller et A. Septier (*) \\ Institut d'Electronique Fondamentale, Bât. 220, Université Paris-XI, 91405 Orsay Cedex, France \\ (*) Laboratoire de Mesures Physiques et Métrologie, CNAM, 292, rue Saint-Martin, 75141 Paris Cedex 03, France
}

(Reçu le 26 mars 1980, révisé le 19 juin 1980, accepté le 23 juin 1980)

\begin{abstract}
Résumé. - Cet article fait suite à une partie théorique traitant le confinement d'ions à l'intérieur d'une cage cylindrique à champ quadrupolaire $\mathrm{HF}$, en vue d'application à l'analyse de gaz résiduels par spectrométrie de masse. La détection des ions confinés est faite par une méthode de résonance qui grâce à sa sélectivité, permet de se placer au point optimum du diagramme de stabilité, ce qui améliore la sensibilité. Une comparaison entre les résultats obtenus avec cette cage et ceux que l'on obtient en utilisant un spectromètre commercial, le quadrupôle simplifié Riber « Miniquade Q-63 » montre les avantages et inconvénients d'utilisation de la cage cylindrique.
\end{abstract}

\begin{abstract}
In a first paper we gave a theoretical treatment of the confinement of ions in a cylindrical trap of quadrupolar symmetry with special application to mass spectrometry. The present paper deals with experimental results. A resonance ion detection system is employed which due to its selectivity, permits the use of the optimum operating point in the stability diagram and so selectivity is improved. A comparison of the results obtained with the trap and those obtained with a commercial spectrometer, the simple Riber quadrupole " Miniquade Q-63» shows the advantages and disadvantages of the cylindrical trap.
\end{abstract}

1. Introduction. - La cage donnant un champ quadrupolaire parfait à trois dimensions comporte des électrodes qui sont des hyperboloïdes de révolution [1] autour de l'axe des $z$. Cette répartition de champ peut être obtenue de façon approchée en utilisant un système simplifié où l'électrode centrale est constituée d'un cylindre creux et les deux électrodes extérieures sont deux disques circulaires. Cette cage a en théorie [2] des propriétés presque identiques à celles de la cage hyperbolique et présente l'avantage essentiel d'être beaucoup plus simple à réaliser.

Elle a été utilisée pour des expériences de la spectroscopie hyperfine des ions piégés [2,3] et comme source d'ions [4], mais une étude comparative de ses performances avec un autre type de spectromètre n'avait jamais été faite à notre connaissance pour l'analyse de gaz résiduels dans une enceinte à ultravide.

Nous décrivons tout d'abord le dispositif expérimental, utilisé pour nos expériences, puis nous comparerons les résultats fournis par notre cage à ceux obtenus avec un quadrupôle Riber, type « Miniquade Q-63 » placé dans la même atmosphère résiduelle.
2. Dispositif expérimental. Détection des ions stockés. - 2.1 ENCEINTE A VIDE. SYSTÈME DE POMPAGE. - La cage cylindrique et le quadrupôle sont fixés respectivement dans deux bras horizontaux identiques d'une enceinte à vide. Une jauge à ionisation est fixée sur un troisième bras pour la mesure du vide. L'étanchéité est assurée par des joints métalliques.

Le groupe de pompage se compose d'une pompe à diffusion d'huile (huile silicone DC 705), couplée à une pompe rotative primaire. Le vide limite dans l'enceinte est de l'ordre de $10^{-9}$ torr, après dégazage des éléments à $200^{\circ} \mathrm{C}$ durant quelques heures.

Un piège à azote liquide condense l'huile provenant de la pompe primaire et un deuxième piège assure la protection de l'enceinte contre les vapeurs d'huile de la pompe secondaire.

Le schéma complet du système de pompage et de l'enceinte à vide est illustré par la figure 1 .

2.2 Dispositif DE STOCKage. LA CAGE. - En pratique, il serait intéressant de pouvoir utiliser des cages de petites dimensions. Mais les dimensions de la 


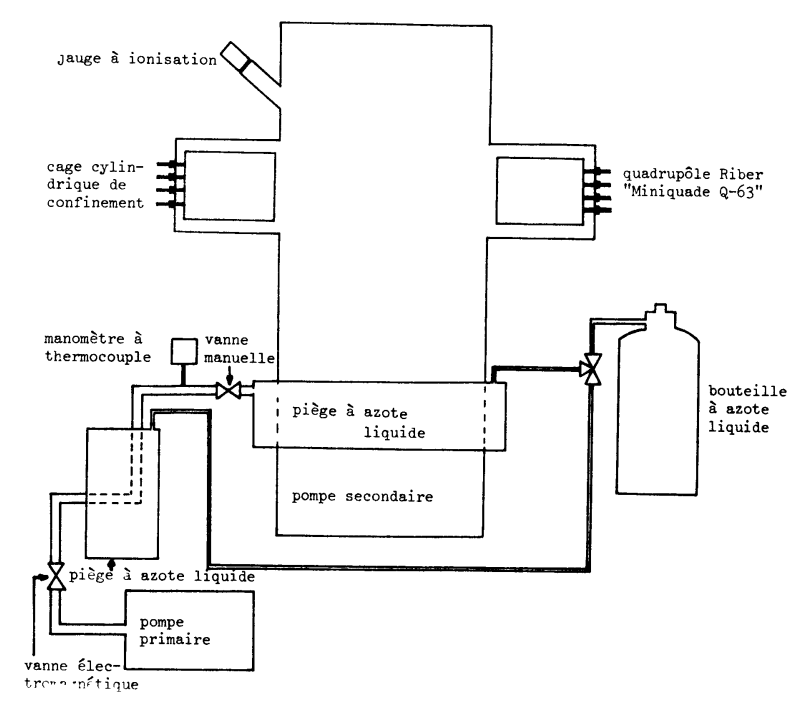

Fig. 1. - Enceinte à vide et système de pompage.

[Vacuum chamber and pumping system.]

cage jouent d'une part sur l'efficacité de confinement, et d'autre part sur la densité maximum d'ions pouvant être confinés.

En fait, le potentiel effectif de confinement vu par les ions et dont l'expression est donnée dans la $1^{\text {re }}$ partie [5], est proportionnel au produit $\left(z_{1} \Omega\right)^{2}$. $\mathrm{Par}$ conséquent, avec une augmentation de $z_{1}$ (ou de $\Omega$ ) on arrive à augmenter ce potentiel effectif (et par conséquent le puits de potentiel vu par les ions [6]) et l'efficacité de confinement est accrue.

Aussi, nous avons vu [5] que la densité maximum d'ions pouvant être confinés augmente également proportionnellement à $\left(z_{1} \Omega\right)^{2}$ et l'influence du champ de charge d'espace parasite diminue. On a donc intérêt à prendre des valeurs de $z_{1}$ et $\Omega$ aussi grandes que possibles.

L'augmentation de la fréquence présente l'intérêt de diminuer l'influence sur le taux de confinement de la vitesse initiale des ions. En effet la vitesse réduite, intervenant dans les équations du mouvement, est donnée comme nous l'avons vu en [5], par les expressions :

$$
\frac{\mathrm{d} r}{\mathrm{~d} \tau}=\frac{\mathrm{d} r}{\mathrm{~d} t} \times \frac{2}{\Omega} \quad \text { et } \frac{\mathrm{d} z}{\mathrm{~d} \tau}=\frac{\mathrm{d} z}{\mathrm{~d} t} \times \frac{2}{\Omega} .
$$

En augmentant $\Omega$ la vitesse initiale réduite diminue.

Compte tenu de ce dernier avantage, nous avons choisi la fréquence de confinement

$$
F_{0}=\Omega / 2 \pi=1 \mathrm{MHz}
$$

de façon à pouvoir négliger l'influence d'une vitesse initiale égale à la vitesse thermique sur la fraction d'ions conservés par la cage, même pour des masses légères, dont la vitesse thermique est élevée. L'influence de la vitesse thermique de l'hydrogène sur le volume utile à la fréquence de $1 \mathrm{MHz}$ a été calculée en (5). Nous avons trouvé qu'elle était négligeable.
Pour une fréquence donnée, l'augmentation des dimensions de la cage correspond à une augmentation du rapport ions confinés/ions créés. En effet, la surface des ellipses donnant dans un plan $(r, z)$ les positions initiales permises, augmente proportionnellement à $z_{1}$ et $r_{1}$. Nous avons vérifié ce point expérimentalement, en mettant deux cages de dimensions respectives $r_{1}=1,9 \mathrm{~cm}$ et $r_{1}=3,8 \mathrm{~cm}$ simultanément dans l'enceinte à vide et en utilisant deux faisceaux électroniques aussi identiques que possible. La fréquence $F_{0}=\Omega / 2 \pi$ a été choisie de sorte que le produit $z_{1} \Omega$ soit le même dans les deux cas, avec une fréquence suffisamment élevée pour que l'influence de la vitesse initiale thermique des ions soit négligeable $\left(F_{1}=2 \mathrm{MHz}\right.$ et $\left.F_{2}=1 \mathrm{MHz}\right)$. Nous avons constaté que la plus grande cage $\left(r_{1}=3,8 \mathrm{~cm}\right)$ a donné des pics ioniques deux fois plus intenses que ceux de la petite cage $\left(r_{1}=1,9 \mathrm{~cm}\right)$.

Il faut cependant se rappeler que si le produit $z_{1} \Omega$ est plus élevé, pour le même point de fonctionnement $A, Q$ à l'intérieur du diagramme de stabilité $\left(\right.$ avec $A=-\frac{8 e U}{m z_{1}^{2} \Omega^{2}}$ et $\left.Q=\frac{4 e}{m} \frac{V}{z_{1}^{2} \Omega^{2}}\right)$ le confinement d'une masse donnée se produit à des tensions $U$ et $V$ plus élevées. On est alors limité dans l'accroissement de $\Omega$ et $z_{1}$ par la tension disponible à la sortie du générateur $\mathrm{HF}$, par les claquages possibles, et par l'accroissement du bruit dans les circuits de mesure, provoqué par le rayonnement de la tension de confinement sur les deux chapeaux de la cage, reliés à la masse à travers le circuit de détection.

Compte tenu de la tension HF disponible pour nos expériences, nous avons finalement choisi pour les dimensions de la cage les valeurs $r_{1}=1,9 \mathrm{~cm}$ et $z_{1}=1,34 \mathrm{~cm}$ (Fig. 2) de façon à pouvoir observer un spectre de masse s'étendant de $m / e=2$ à $m / e=90$.

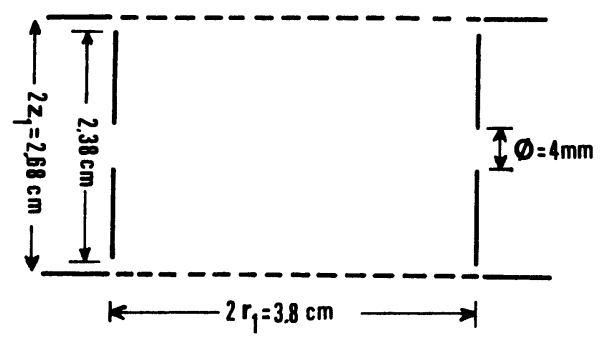

Fig. 2. - Dimensions de la cage de confinement utilisée.

[Dimensions of the trap used in our experiments.]

La tension de confinement, composée d'une tension alternative $V_{\mathrm{a}}=V \cos \Omega t$ à la fréquence $F_{0}=1 \mathrm{MHz}$ et d'une tension continue $U$ obtenue par redressement d'une tension alternative proportionnelle à $V_{\mathrm{a}}$, est appliquée sur l'électrode centrale cylindrique de la cage et les deux électrodes extérieures, appelées chapeaux, sont reliées à la masse à travers le circuit de détection.

2.3 SChéma ÉleCtRIQUE : SYSTÈme DE DÉTECTION. GÉNÉRATEUR DE LA TENSION DE CONFINEMENT. - La 


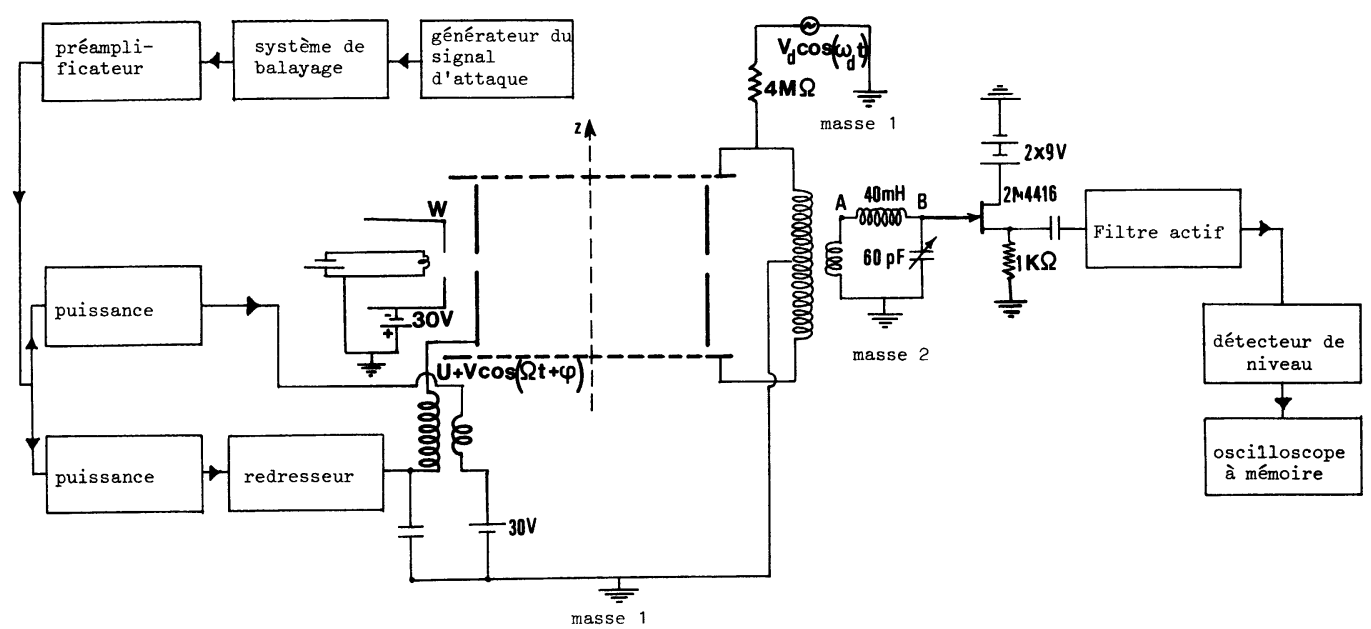

Fig. 3. - Schéma synoptique du générateur de la tension $U+V \cos (\Omega t)$, appliquée sur l'électrode centrale de la cage, du système de détection et du canon à électrons.

[Schematic representation of the r.f.-d.c. generator, the detection system and the electron gun.]

méthode de détection utilisée (détection par absorption résonnante d'énergie) est une méthode sélective, permettant de se placer au point optimum du diagramme de stabilité et ainsi avoir un meilleur rendement de confinement,

Pour des valeurs données de $A$ et $Q$ à l'intérieur du diagramme de stabilité, les ions confinés oscillent à l'intérieur de la cage à des fréquences fondamentales $f_{r}$ et $f_{z}$, caractéristiques de la masse et de l'état de charge de l'ion. Une bobine de coefficient de qualité élevé est branchée entre les deux chapeaux et l'ensemble des deux chapeaux et de la bobine de détection constitue un circuit oscillant. Ce circuit est excité par un générateur de tension à travers une résistance de $4 \mathrm{M} \Omega$ à la fréquence fixe $f_{\mathrm{d}}$. En balayant les tensions $U$ et $V$ de confinement, on arrive à imposer successivement aux différentes masses la fréquence d'oscillation $f_{z}=f_{\mathrm{d}}$ et au moment où les oscillations des ions à l'intérieur de la cage, et la tension aux bornes du circuit LC extérieur sont en résonance, un échange d'énergie se produit et une chute de tension apparaît aux bornes de ce circuit.

Sur la figure 3 on donne le schéma du système de détection, et du système fournissant la tension de confinement.

La tension de confinement à la fréquence de $1 \mathrm{MHz}$ étant balayée de zéro à $200 \mathrm{~V}$, un signal parasite apparaît sur les chapeaux de la cage, reliées à la masse à travers le circuit de détection d'impédance non nulle. Afin d'améliorer le rapport signal utile/signal parasite, le point milieu de la bobine de détection est relié à la masse et un système de transformateur est utilisé. A l'aide d'un filtre LC passe-bande, branché à la sortie du secondaire du transformateur et accordé à la fréquence de résonance de la bobine, constituant le primaire, on améliore encore le rapport signal utile/ signal parasite.

Un transistor à effet de champ à faible bruit assure l'adaptation d'impédance avec le reste du montage et un filtre actif sert à éliminer au maximum la tension HF parasite résiduelle. La chute de tension à la résonance est détectée à l'aide d'un détecteur de niveau TEKELEC TE9822 et le spectre est observé soit sur un oscilloscope à mémoire, soit sur une table traçante.

Le balayage pour l'obtention du spectre se fait de la façon suivante : les fréquences $F_{0}$ de confinement et $f_{\mathrm{d}}$ du circuit oscillant de détection sont maintenues constantes et les tensions $U$ et $V$ sont balayées linéairement, tout en gardant leur rapport $V / U$ constant. $\mathrm{Ce}$ mode de balayage a un double avantage : il est simple à réaliser, et à toutes les masses observées correspond le même point $A, Q$ du diagramme de stabilité, ce qui permet une étude comparative de différents pics

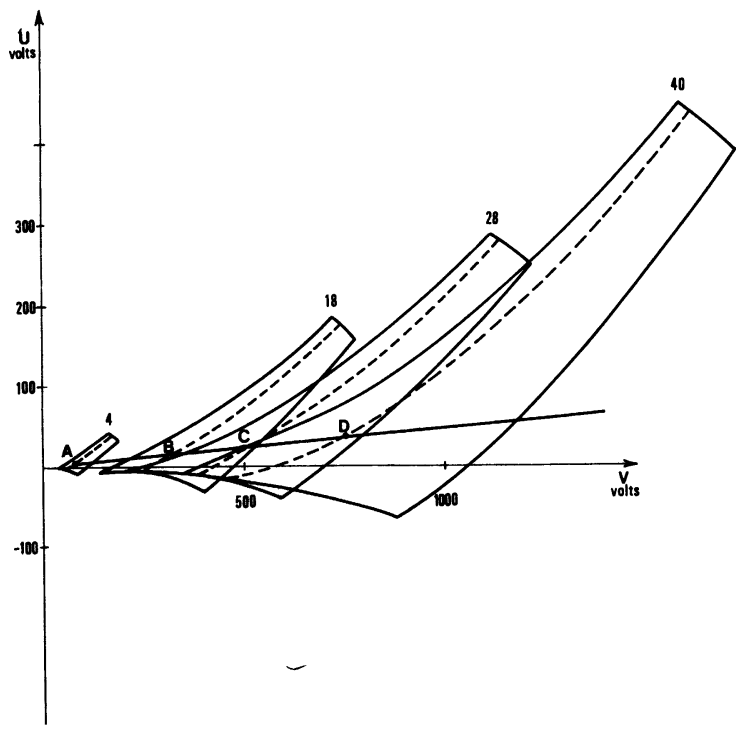

Fig. 4. - Droite de balayage utilisée. L'intersection de la droite de balayage avec la courbe iso- $\beta_{z}$ choisie (pour nos expériences $\beta_{z}=0,32$ ) donne les tensions $U$ et $V$ pour lesquelles on obtient les différents pics.

[Operating line used. The intersection of the operating line with the chosen iso- $\beta_{z}$ line (for our experiences $\beta_{z}=0.32$ ) gives the voltages $U$ and $V$ corresponding to the different pics.] 
observés. La droite de balayage utilisée est tracée sur la figure 4 dans les diagrammes $U, V$ correspondant aux ions de $m / e=4, m / e=18, m / e=28$, et $m / e=40$. L'intersection de la droite de balayage avec la courbe $\beta_{z}$ utilisée, tracée à l'intérieur de chaque diagramme, donne théoriquement les tensions d'observation des pics correspondants (points A, B, C, D). Expérimentalement les pics sont observés pour des tensions $U$ et $V$ plus élevées que celles obtenues théoriquement. $\mathrm{Ce}$ déplacement peut être dû soit à une imprécision du calcul de la courbe $\beta_{z}$ théorique, soit, pour des pressions élevées, à la présence d'une charge d'espace ionique importante, correspondant à une tension continue supplémentaire, qui déplace le point de fonctionnement $A, Q$ à l'intérieur du diagramme de stabilité.

Afin de pouvoir faire un balayage simultané des deux composantes, continue et alternative, de la tension de confinement, on utilise un préamplificateur qui attaque simultanément deux étages (Fig. 3), un étage de puissance, fournissant la tension $\mathrm{HF}$, et un deuxième étage qui fournit, après redressement, la composante continue. Le balayage des composantes $U$ et $V$ se fait alors en balayant la tension alternative à l'entrée du préamplificateur, soit automatiquement à l'aide d'une tension en dents de scie et un système modulateur comportant un multiplicateur Motorola MC 15951 en combinaison avec l'amplificateur opérationnel LM 733, soit manuellement à l'aide d'un potentiomètre.

La production des ions à l'intérieur de la cage est obtenue grâce à un faisceau électronique, dirigé suivant l'axe des $r$ de la cage. La cathode, en fil de tungstène Rhénié, est reliée à la masse et un wehnelt, porté à un potentiel légèrement négatif, focalise les électrons du faisceau (Fig. 3). Ces électrons sont accélérés par la tension de confinement appliquée sur l'électrode centrale de la cage, pendant les demi-périodes où celle-ci est positive. Un trou de $4 \mathrm{~mm}$ de diamètre est percé dans l'électrode centrale pour le passage du faisceau et un deuxième trou, diamétralement opposé au précédent permet la sortie des électrons.

2.4 Signal IONIQUe. - L'amplitude du signal observé lorsque les oscillations des ions à l'intérieur de la cage et la tension aux bornes du circuit LC entrent en résonance, a été calculée de façon approchée dans les références [6] et [7]. Elle est donnée par l'expression suivante :

$$
V_{\text {ion. }}=\frac{N Z e Q_{0}}{4 C}
$$

où $N$ est le nombre d'ions oscillant en résonance avec le circuit extérieur, $Z e$ l'état de charge de ces ions, $Q_{0}$ le coefficient de surtension du circuit oscillant et $C$ sa capacité parallèle (capacité entre les deux chapeaux considérés comme un condensateur plan, et capacité des câbles de branchement).
La méthode de calcul utilisée pour l'évaluation du signal ionique, souffre des inconvénients suivants :

- Elle considère un champ électrique constant appliqué entre les deux chapeaux pour mettre en cohérence le mouvement ionique. Mais lorsqu'on est à la résonance la chute de tension due à la présence des ions fait varier ce champ. Si le nombre d'ions à l'intérieur de la cage est élevé, cette chute de tension peut être importante, et il faudrait alors en tenir compte pour obtenir une expression plus précise du signal ionique.

- Le champ dipolaire de détection modifie les trajectoires ioniques à l'intérieur de la cage, comme nous le verrons dans le paragraphe 2.6. Cette modification est différente selon la tension $V_{\mathrm{d}}$ appliquée et selon le rapport $m / Z e$ des ions. Il faudrait également en tenir compte pour l'évaluation du signal ionique observé.

- Lorsque le nombre d'ions à l'intérieur de la cage augmente, on constate expérimentalement une saturation du signal ionique, si la tension $V_{\mathrm{d}}$ de détection reste constante. Le champ dipolaire appliqué n'est pas suffisant pour mettre en cohérence le plus grand nombre d'ions créés lorsque la pression augmente. Par contre en augmentant $V_{\mathrm{d}}$, on augmente également le signal ionique observé. Donc le signal de résonance peut être proportionnel au nombre d'ions oscillant à l'intérieur de la cage uniquement lorsque la tension de détection augmente avec la pression. L'application d'une tension $V_{\mathrm{d}}$ élevée pour des faibles pressions n'est pas indiquée à cause de son effet destructif sur les trajectoires ioniques (voir $\S 2.6$ ).

- La vitesse de balayage des tensions $U$ et $V$ détermine le temps pendant lequel le point de fonctionnement correspondant à une certaine espèce ionique, reste à l'intérieur du diagramme de stabilité $(A, Q)$. Elle détermine donc le temps pendant lequel les ions créés par le faisceau électronique sont confinés. Cette vitesse de balayage devrait donc intervenir aussi dans un calcul précis du signal ionique observé. En outre, nous avons vu dans la partie théorique [5] que les imperfections du champ quadrupolaire entraînent une variation de la fréquence fondamentale de l'oscillation des ions en fonction de leur position initiale. Les ions de même rapport $m / Z e$ n'oscillent donc pas tous à la même fréquence, ce qui va se traduire par un élargissement de la raie observée, et une diminution de son amplitude. L'interaction des ions avec le champ de détection est donc plus compliquée que dans le cas d'un champ quadrupolaire parfait. A cause de tous ces facteurs, on peut seulement affirmer que l'amplitude du signal observé doit être lié au nombre d'ions oscillant à l'intérieur de la cage par une expression beaucoup plus compliquée que (1). Toutefois, l'expérience montre qu'à des pressions partielles inférieures à $10^{-8}$ torr, le signal ionique est proportionnel à la pression partielle du gaz observé et donc en première approximation au nombre d'ions créés dans la cage. Nous avons aussi vérifié expérimentalement que le 
signal ionique observé est proportionnel au rapport $Q_{0} / C$ du coefficient de surtension du circuit oscillant à sa capacité parallèle $C$.

2.5 Signal minimum Détectable. Signal MAXIMUM. - Le signal minimum détectable, en l'absence de tout signal parasite, serait limité par la tension de bruit thermique dans la résistance parallèle du circuit LC. Cette tension de bruit est donnée par la formule de Nyquist :

$$
e_{\mathrm{b}}=\sqrt{\bar{V}_{\mathrm{b}}^{2}}=\sqrt{4 K T R \Delta f_{z}}
$$

où $\Delta f_{z}$ est la bande passante du circuit oscillant. En tenant compte des expressions :

$$
R=\frac{Q_{0}}{\omega_{z} C} \quad \text { et } \quad Q_{0}=\frac{f_{z}}{\Delta f_{z}}
$$

la formule (2) prend la forme :

$$
e_{\mathrm{b}}=\sqrt{\frac{4 K T}{2 \pi C}} .
$$

Donc en diminuant la capacité $C$, on augmente, selon la relation (1) le signal utile, mais on augmente en même temps le bruit thermique (relation 3). Heureusement le facteur d'accroissement est plus faible dans le deuxième cas, et il peut être avantageux de diminuer $C$.

Les caractéristiques du circuit oscillant que nous avons utilisé étaient les suivantes :

$$
\begin{gathered}
f_{z}=160 \mathrm{KHz}, \quad R=2 \mathrm{M} \Omega, \Delta f_{z}=2,67 \mathrm{KHz}, \\
Q_{0}=60 \text { et } C=30 \mathrm{pF} .
\end{gathered}
$$

D'après l'expression (2) ceci correspond à un signal minimum détectable de $9 \mu \mathrm{V}$ aux bornes du circuit oscillant. A partir de l'expression (1) on trouve un nombre minimum d'ions détectable de $N=110$, cette sensibilité limite de la méthode de détection par résonance pouvant encore être améliorée en utilisant un circuit oscillant de meilleur rapport $Q_{0} / C$.

$\mathrm{Si}$ on s'intéresse maintenant aux fortes populations ioniques, la chute de tension maximum que l'on peut obtenir à cause de la présence des ions, ne peut pas dépasser la tension $V_{\mathrm{d}}$, appliquée pour exciter le circuit résonnant. A cause de l'effet destructif de la tension $V_{\mathrm{d}}$ sur le confinement (voir $\$ 2.6$ ), cette tension est limitée à quelques $\mathrm{mV}$ sur les chapeaux. En augmentant le rapport $Q_{0} / C$, on augmente la sensibilité du système de détection mais la chute de tension due aux ions atteint rapidement la valeur limite. Il faut donc amortir le circuit LC lorsque la pression augmente pour éviter la saturation du système de détection.

Avec le circuit oscillant utilisé dans nos expériences $\left(Q_{0}=60\right.$ et $\left.C=30 \mathrm{pF}\right)$ le signal ionique est toujours resté inférieur au signal $V_{\mathrm{d}}$ appliqué, même à des pressions partielles de l'ordre de $10^{-5}$ torr, et on n'a pas observé de saturation due à cet effet.
2.6 INFLUENCE DU CHAMP DIPOLAIRE DE DÉTECTION SUR LES TRAJECTOIRES IONIQUES. - La tension excitatrice $V_{\mathrm{d}}$, appliquée sur un chapeau à travers la résistance $R$, crée entre les deux chapeaux un champ donné en première approximation par :

$$
\mathbf{E}_{\text {det. }}=-\frac{2 V_{\mathrm{d}} \cos \left(\omega_{\mathrm{d}} t+\varphi_{1}\right)}{2 z_{1}} \hat{\mathbf{z}}
$$

(expression valable en assimilant l'ensemble des deux chapeaux à un condensateur plan) :

$2 z_{1}$ est la distance entre les deux chapeaux, $\omega_{\mathrm{d}}$ la fréquence d'oscillation du circuit résonnant et $\hat{\mathbf{z}}$ le vecteur unitaire dans la direction $z$.

Ce champ s'ajoute à la composante du champ quadrupolaire dans la direction $z$ et le champ total dans cette direction devient :

$$
\begin{array}{r}
E_{z}=\sum_{n}(-1)^{n} \frac{2 V_{0} I_{0}\left(p_{n} r\right) \sin \left(p_{n} z\right)}{z_{1} I_{0}\left(p_{n} r_{1}\right)}- \\
-\frac{2 V_{\mathrm{d}} \cos \left(\omega_{\mathrm{d}} t+\varphi_{1}\right)}{2 z_{1}}
\end{array}
$$

où

$$
V_{0}=U+V \cos (\Omega t+\varphi)
$$

En utilisant les paramètres réduits :

$$
A=-\frac{8 e U}{m z_{1}^{2} \Omega^{2}}, \quad Q=\frac{4 e V}{m z_{1}^{2} \Omega^{2}}
$$

et en définissant les deux nouveaux paramètres :

$$
B=-\frac{4 e V_{\mathrm{d}}}{m z_{1}^{2} \Omega^{2}} \quad \text { et } \quad A C=\frac{2 \omega_{\mathrm{d}}}{\Omega}
$$

on arrive à la forme suivante de la composante du mouvement dans la direction $z$ :

$$
\begin{aligned}
\frac{\mathrm{d}^{2} z}{\mathrm{~d} \tau^{2}}= & -\{A-2 Q \cos (2 \tau+\varphi)\} \sum_{n}(-1)^{n} \times \\
& \times \frac{z_{1} I_{0}\left(p_{n} r\right) \sin \left(p_{n} z\right)}{I_{0}\left(p_{n} r_{1}\right)}+B \cos \left(A C \cdot \tau+\varphi_{1}\right)
\end{aligned}
$$

En pratique, la tension de détection appliquée est de l'ordre de quelques $\mathrm{mV}$ aux bornes du circuit oscillant. En introduisant le terme supplémentaire dans les équations du mouvement, avec $V_{d}=10 \mathrm{mV}$, on n'a observé aucune influence sur le mouvement ionique, au moins pendant les premières dizaines de périodes du champ HF. Par contre, si nous considérons une tension de détection nettement plus forte : $V_{\mathrm{d}}=1 \mathrm{~V}$, avec $\varphi_{1}=0, \Omega=2 \pi \times 10^{6} \mathrm{rad} / \mathrm{s}$ et $m / Z e=28$, donc $B=-0,000026$, nous obtenons les résultats suivants :

- Pour le point de fonctionnement $A=-0,1$, $Q=1,3$, si $A C=0,5067$, donc $A C=\beta_{z}$ (la fréquence du circuit oscillant extérieur coïncide avec la fréquence d'oscillation des ions), l'amplitude de la trajectoire en $z$ augmente et la trajectoire tend à devenir instable (Fig. 5). 

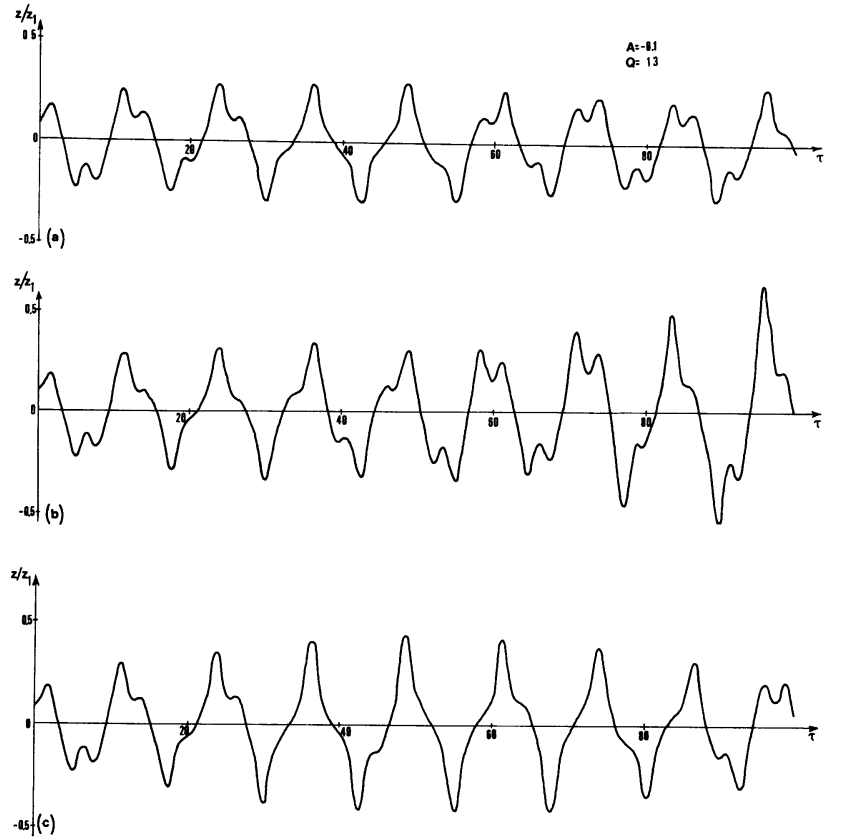

Fig. 5. - Variation de $z / z_{1}$ du mouvement d'un ion en fonction de $\tau=\Omega t / 2$ (vitesse initiale et phase initiale nulles) $: a$ ) en absence de champ dipolaire $\left.\left(\beta_{z}=0,5067\right) ; b\right)$ en présence d'un champ dipolaire de fréquence $f_{\mathrm{d}}$ égale à la fréquence fondamentale du mouvement ionique en $\left.z\left(A C=\beta_{z}=0,5067\right) ; c\right)$ en présence d'un champ dipolaire dont la fréquence est voisine de la fréquence fondamentale du mouvement ionique en $z(A C=0,46)$.

[Variation of $z / z_{1}$ of an ion mouvement as a function of $\tau=\Omega t / 2$ (zero initial velocity and zero initial phase) $: a$ ) in the absence of a detection field $\left.\left(\beta_{z}=0.5067\right) ; b\right)$ in the presence of a detection field of frequency $f_{\mathrm{d}}$ equal to the fundamental frequency of the ionic mouvement $\left.\left(A C=\beta_{z}=0.5067\right) ; c\right)$ in the presence of a detection field whose frequency approches the fundamental frequency of the ionic motion in the $z$ direction $(A C=0.46)$.]

- Si les ions oscillent à une fréquence voisine de celle du circuit extérieur (par exemple $A C=0,46$ ), le champ de détection agit sur les trajectoires ioniques et entraîne une augmentation de l'amplitude de la trajectoire par rapport au cas où le champ dipolaire n'existe pas, mais la trajectoire reste stable. Cette augmentation d'amplitude devient d'autant plus faible que la fréquence d'oscillation des ions s'éloigne de la fréquence du circuit extérieur.

Le calcul effectué nous montre donc l'effet destructif de la tension de détection sur les ions confinés et la nécessité d'utiliser des tensions excitatrices aussi faibles que possible.

Avec des tensions de détection faibles (de l'ordre de quelques dizaines de $\mathrm{mV}$ ), l'auǵmentation d'amplitude de la trajectoire à la résonance est lente et la trajectoire devient instable après un temps plus long que quelques dizaines de périodes HF. En pratique, nous avons constaté que la tension de détection est de l'ordre de quelques $\mathrm{mV}$ pour des pressions partielles inférieures à $10^{-8}$ torr. Pour des masses légères il faut utiliser des tensions de détection encore plus faibles (le paramètre $B$ du terme supplémentaire est inversement proportionnel à la masse).
3. Résultats expérimentaux. Comparaison entre résultats obtenus respectivement avec la cage et avec le quadrupôle Riber "Miniquade Q-63». - Nous avons fixé pour notre cage les fréquences respectives de confinement et de détection à $F_{0}=1 \mathrm{MHz}$ et $f_{\mathrm{d}}=160 \mathrm{kHz}$.

Le quadrupôle Riber Q-63, utilisé comme élément de comparaison, comporte [8] une source d'ions, où les ions sont créés par bombardement électronique, et un filtre de masse quadrupolaire de longueur $z_{0}^{\prime}=5 \mathrm{~cm}$ et de rayon intérieur inter-électrode $r_{0}^{\prime}=0,25 \mathrm{~cm}$ pour leur séparation selon leur rapport $m / Z e$. Les électrodes du filtre sont 4 cylindres placés parallèlement, de sorte que leur rayon $\alpha^{\prime}$ et le rayon du cercle tangent 4 cylindres satisfont la relation :

$$
\alpha^{\prime}=1,16 r_{0}^{\prime} \text {. }
$$

Dans le filtre quadrupolaire les ions sont confinés seulement dans les directions $x$ et $y$, et se déplacent suivant $z$ avec une vitesse constante. Les ions sortant du filtre sont collectés et le courant est amplifié par un préamplificateur à transistors MOS. Après amplification supplémentaire, le spectre est obtenu soit sur un oscilloscope à mémoire, soit sur un enregistreur. La pression minimale détectable est de $5 \times 10^{-12}$ torr. Un galvanomètre (gradué en torr de $\mathrm{N}_{2}$ ), placé sur la face avant de l'alimentation du quadrupôle, donne directement, dans la limite de précision de l'appareil, des pressions partielles en équivalent azote.

La pression totale maximale d'utilisation du spectromètre est de $10^{-3}$ torr. Le spectre balayé s'étend de $m / e=1$ à $m / e=80$ en une seule gamme.

La source d'ions et le filtre quadrupolaire sont montés sur une bride standard de $\varnothing 38 \mathrm{~mm}$ pour ultravide.

Dans le quadrupôle Riber, l'énergie des électrons ionisants est de $75 \mathrm{eV}$ et les facteurs de correction de la hauteur relative des pics, qui rendent compte des sections efficaces d'ionisation différentes, sont donnés pour quelques gaz sur le tableau I. Sur le même tableau, on donne les facteurs de correction des pics de ces mêmes ions créés à l'intérieur de la cage. Ces facteurs ont été calculés [5] pour le point de fonctionnement $A=-0,1$ et $Q=1,3$ et pour le cas d'une ionisation radiale avec la cathode du canon à électrons reliée à la masse.

A des pressions élevées, la charge d'espace ionique, accumulée à l'intérieur de la cage, crée un champ électrique supplémentaire qui s'oppose au confinement d'autres ions et limite le nombre final d'ions pouvant être piégés. L'effet des collisions des ions avec les neutres existant à l'intérieur de la cage, joue également un rôle destructif sur le confinement lorsque la pression augmente. Le fonctionnement du quadrupôle Riber n'étant pas basé sur un confinement des ions dans un volume de dimensions finies, ces phénomènes $\mathrm{y}$ sont beaucoup moins gênants. 
Tableau I

\begin{tabular}{ccccc} 
& \multicolumn{2}{c}{ Quadrupôle Riber } & \multicolumn{2}{c}{ Cage cylindrique } \\
Elément & $\begin{array}{c}\sigma_{01} \\
\left(\mathrm{~cm}^{2}\right)\end{array}$ & $\begin{array}{c}\text { Facteur de } \\
\text { correction }\end{array}$ & $\begin{array}{c}\overline{\sigma_{01} F_{r}} \\
\left(\mathrm{~cm}^{2}\right)\end{array}$ & $\begin{array}{c}\text { Facteur de } \\
\text { correction }\end{array}$ \\
- & - & - & - & - \\
$\mathrm{He}$ & $0,38 \times 10^{-16}$ & 7,25 & $6,8 \times 10^{-18}$ & 5,44 \\
$\mathrm{H}_{2} \mathrm{O}$ & $2,96 \times 10^{-16}$ & 0,93 & $6,1 \times 10^{-17}$ & 0,61 \\
$\mathrm{~N}_{2}$ & $2,75 \times 10^{-16}$ & 1 & $3,7 \times 10^{-17}$ & 1 \\
$\mathrm{~A}$ & $3,52 \times 10^{-16}$ & 0,78 & $8,3 \times 10^{-17}$ & 0,45
\end{tabular}

$\sigma_{01}$ (deuxième colonne) est la section efficace d'ionisation (passage de neutres aux ions une fois chargés) pour une énergie des électrons ionisants de $75 \mathrm{eV}$.

$\overline{\bar{\sigma}_{01} F_{r}}$ (quatrième colonne) est en moyenne le rapport d'ions confinés/neutres à l'intérieur de la cage [5] pour le point de fonctionnement $A=-0,1, Q=1,3$.

3.1 EFFET DES COLLISIONS SUR LE NOMBRE D'IONS CONFINÉS A L'INTÉRIEUR DE LA CAGE. - L'effet des collisions d'un ion avec des particules existant dans l'atmosphère résiduelle dépend du rapport de la masse de l'ion à la masse de la particule neutre intervenant dans la collision.

Selon la théorie élaborée par Dehmelt et Major [6] et développée par André [9], les collisions d'un ion avec des particules plus légères que lui, conduisent à une diminution progressive de l'énergie des ions ce qui favorise le rendement de piégeage. L'amélioration est d'autant plus efficace que les particules neutres sont plus légères.

Si par contre un ion subit des collisions avec des particules plus lourdes que lui, les collisions conduisent à une augmentation de l'énergie des ions et donc à une diminution du rendement de piégeage. Si la population ionique piégée est hétérogène, les ions légers seront donc défavorisés par rapport aux ions lourds à cause

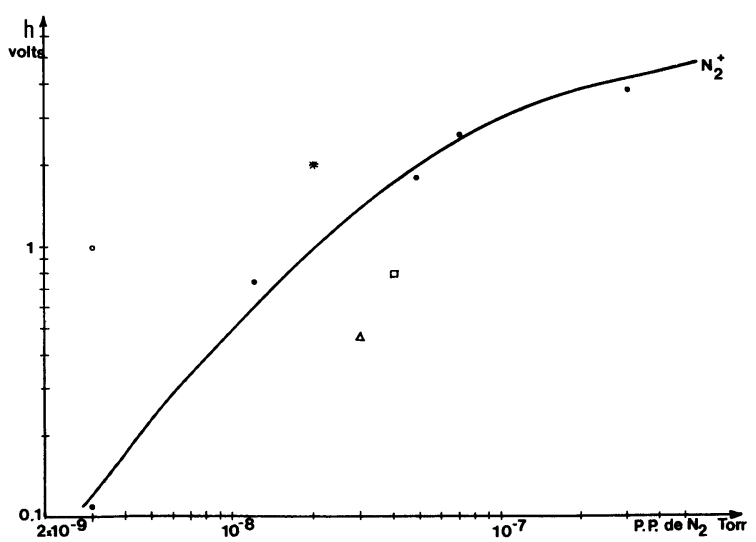

Fig. 6. - - Hauteur des pics de l'ion $\mathrm{N}_{2}^{+}$en fonction de la pression partielle d'azote injecté extérieurement ; $\Delta$ hauteur du signal $\mathrm{N}_{2}^{+}$ en présence d'une pression partielle d'argon de $1,15 \times 10^{-7}$ torr $\square$ signal de $\mathrm{N}_{2}^{+}$en présence de $1,3 \times 10^{-7}$ torr d'argon ; * signa de $\mathrm{N}_{2}^{+}$en présence de $2,5 \times 10^{-5}$ torr d'hélium; $\mathrm{O}$ signal de $\mathrm{N}_{2}^{+}$ en présence de $7,25 \times 10^{-6}$ torr d'hélium.

[- Ionic signal corresponding to $\mathrm{N}_{2}^{+}$as a function of the partial pressure of nitrogen injected externally; $\Delta$ signal of $\mathrm{N}_{2}^{+}$in the presence of $1.15 \times 10^{-7}$ torr of argon $; \square$ signal of $\mathrm{N}_{2}^{+}$in the presence of $1.3 \times 10^{-7}$ torr of argon; $*$ signal of $\mathrm{N}_{2}^{+}$in the presence of $2.5 \times 10^{-5}$ torr of helium; $O$ signal of $\mathrm{N}_{2}^{+}$in the presence of $7.25 \times 10^{-6}$ torr of helium.] de l'effet des collisions sur les atomes ou molécules $\mathrm{du}$ gaz résiduel.

Pour vérifier l'influence des collisions sur le rendement du confinement (taux d'ions confinés par rapport à ceux qui sont créés), nous avons effectué l'expérience suivante :

Dans une atmosphère résiduelle à la pression globale de $5 \times 10^{-9}$ torr, nous avons fait une injection progressive d'azote et nous avons tracé la variation de la hauteur du pic correspondant à l'ion $\mathrm{N}_{2}^{+}$en fonction de la pression partielle de son gaz parent, mesurée à l'aide du quadrupôle Riber. La courbe correspondante représentée figure 6 servira de référence pour les mesures suivantes.

Nous avons ensuite arrêté l'injection d'azote et lorsque le vide limite était de nouveau atteint, nous avons procédé à une injection d'argon. La pression partielle d'argon était mesurée à l'aide du quadrupôle Riber, en tenant compte du facteur de correction correspondant. Nous avons de nouveau mesuré la hauteur du pic de l'ion $\mathrm{N}_{2}^{+}(m / e=28)$ en vérifiant la pression partielle de l'azote. En présence d'une pression partielle importante d'argon $(m / e=40)$, la même pression partielle d'azote donne des pics plus faibles. Par exemple sur le spectre de la figure 7, obtenu en

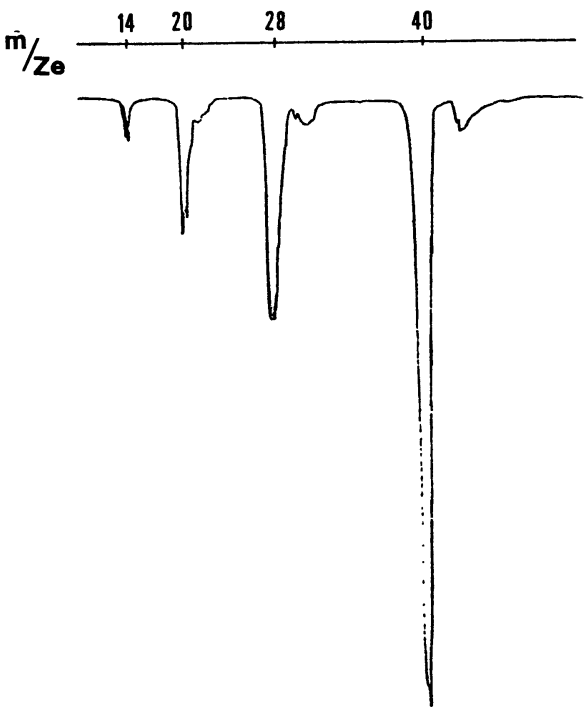

Fig. 7. - Spectre obtenu avec la cage en présence d'argon et d'azote, injectés extérieurement. La pression partielle d'argon était $1,3 \times 10^{-7}$ torr et celle de l'azote : $4 \times 10^{-8}$ torr. Sensibilité verticale de l'enregistreur $200 \mathrm{mV} / \mathrm{cm}$.

[Spectrum obtained with the cylindrical trap in the presence of argon and nitrogen, injected externally. The partial pressure of argon was $1.3 \times 10^{-7}$ torr and that of nitrogen : $4 \times 10^{-8}$ torr.]

présence d'argon sous une pression partielle de $1,3 \times 10^{-7}$ torr, à l'ion $\mathrm{N}_{2}^{+}$correspond un pic de $0,72 \mathrm{~V}$ pour une pression partielle de $4 \times 10^{-8}$ torr d'azote. En l'absence d'argon, la même pression partielle d'azote conduit à un pic de 1,6 V (Fig. 6). La présence des atomes d'argon joue un rôle destructif sur les ions $\mathrm{N}_{2}^{+}$d'une part à cause des collisions des 
ions $\mathrm{N}_{2}^{+}$avec les atomes d'argon, d'autre part à cause de la présence d'une charge d'espace ionique plus élevée, les ions $\mathrm{A}^{+}$étant eux-mêmes piégés pendant l'observation du pic 28.

La même expérience a été faite également avec l'hélium $(m / e=4)$. La présence d'une pression partielle d'hélium allant de quelques $10^{-6}$ à $10^{-5}$ torr conduit à une augmentation de la hauteur du pic de l'azote. Les résultats obtenus sont donnés sur la figure 6. Cette expérience confirme bien une amélioration du confinement, qui se traduit par une augmentation du signal ionique, due aux collisions avec le gaz léger.
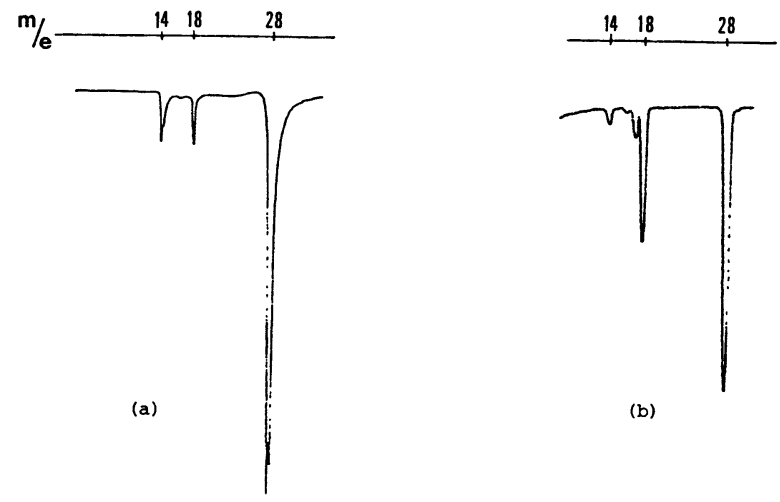

Fig. 8. - Les spectres $(a)$ et $(b)$ ont été obtenus en présence d'une pression partielle d'hélium de $3,4 \times 10^{-6}$ torr. La pression partielle d'azote à l'intérieur de l'enceinte était de $1,6 \times 10^{-8}$ torr. $a$ ) Cage : sensibilité verticale de l'enregistreur : $200 \mathrm{mV} / \mathrm{cm} ; b$ ) Quadrupôle Riber : sensibilité verticale de l'enregistreur : $20 \mathrm{mV} / \mathrm{cm}$.

[Spectra $(a)$ and $(b)$ were obtained in the presence of a partial pressure of helium of $3.4 \times 10^{-6}$ torr. The partial pressure of argon was $1.6 \times 10^{-8}$ torr. $a$ ) Cylindrical trap : vertical sensibility of the $x$ - $y$ recorder $: 200 \mathrm{mV} / \mathrm{cm} ; b$ ) Riber quadrupole : vertical sensibility of the $x-y$ recorder : $20 \mathrm{mV} / \mathrm{cm}$.]

Sur la figure $8 a$, nous avons donné un spectre obtenu avec la cage en présence d'une pression partielle d'hélium de $3,4 \times 10^{-6}$ torr. La pression partielle de l'azote à l'intérieur de l'enceinte était de $1,6 \times 10^{-8}$ torr. La hauteur du pic correspondant à l'ion $\mathrm{N}_{2}^{+}$était de 1,5 V. En l'absence d'hélium la même pression partielle d'azote conduit, selon la courbe de la figure 6 , à un pic de $0,8 \mathrm{~V}$. Sur la figure $8 b$ nous avons donné un spectre obtenu avec le quadrupôle Riber dans les mêmes conditions que plus haut.

Cette étude comparative confirme l'importance de l'effet des collisions sur le confinement d'ions à l'intérieur de la cage à des pressions élevées. Pour le quadrupôle, l'effet des collisions des ions avec les neutres est négligeable. En fait, les ions traversent le filtre dans la direction $z$ et la distance parcourue entre l'entrée du filtre et le collecteur est à peu près égale à $5,5 \mathrm{~cm}$. Le libre parcours moyen des molécules d'azote à $300 \mathrm{~K}$ est de l'ordre de quelques mètres à une pression de $10^{-5}$ torr, donc bien supérieur à la distance parcourue par les ions.
3.2 VARIATION DE LA HAUTEUR DES PICS DES IONS $\mathrm{N}_{2}^{+}, \mathrm{N}^{+}, \mathrm{A}^{+}$et $\mathrm{A}^{++}$EN FONCTION DE LA PRESSION DE LEUR GAZ PARENT. - Dans toutes les expériences le vide limite était de $5 \times 10^{-9}$ torr.

a) Nous avons tracé la variation de la hauteur des pics des ions de $m / e=28\left(\mathrm{~N}_{2}^{+}\right)$et $m / e=14\left(\mathrm{~N}^{+}\right)$en fonction de la pression partielle d'azote. Les résultats sont donnés sur la figure 9.

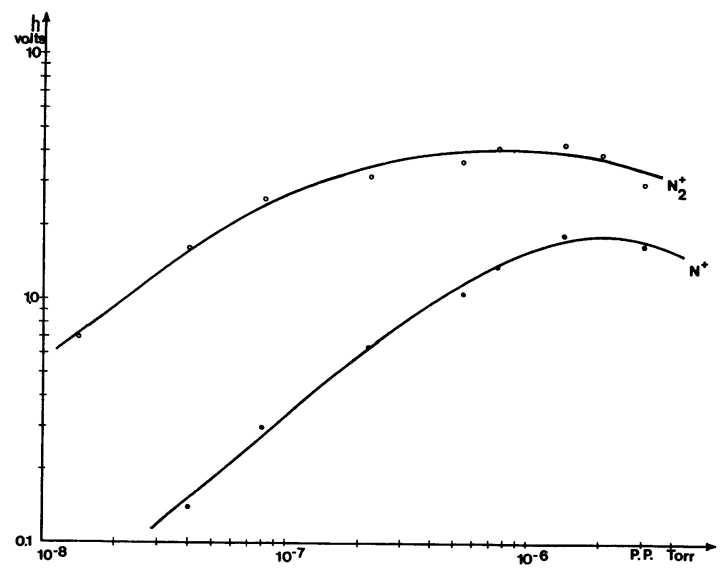

Fig. 9. - Variation de la hauteur des pics des ions $\mathrm{N}_{2}^{+}(m / e=28)$ et $\mathrm{N}^{+}(m / e=14)$ en fonction de la pression d'azote injecté extérieurement.

[Variation of the signal corresponding to $\mathrm{N}_{2}^{+}(m / e=28)$ and $\mathrm{N}^{+}$ $(m / e=14)$ as a function of the partial pressure of nitrogen.]

Les deux courbes correspondant aux ions $\mathrm{N}_{2}^{+}$et $\mathrm{N}^{+}$ respectivement, présentent d'abord une partie linéaire pour les faibles pressions partielles d'azote ; par contre, pour des pressions plus élevées, une saturation est progressivement atteinte. Pour les ions $\mathrm{N}_{2}^{+}$cette saturation est atteinte à une pression partielle $\therefore$ azote plus faible que pour les ions $\mathrm{N}^{+}$, mais le signal de saturation est plus élevé pour les ions $\mathrm{N}_{2}^{+}$. Enfin, à des pressions encore plus élevées, l'amplitude des pics diminue.

Les phénomènes suivants peuvent être envisagés pour expliquer la saturation du signal ionique :

- Lorsque le nombre total d'ions piégés à l'intérieur de la cage augmente, si la tension de détection reste constante, à partir d'une certaine valeur la réponse du système de détection n'est plus une fonction linéaire du nombre d'ions piégés, donnée par l'expression simple (1). Le champ dipolaire de détection n'est pas suffisamment élevé pour mettre en mouvement cohérent l'ensemble des ions, et seulement une partie de ces ions se mettent en interaction avec le circuit oscillant. Egalement, la chute de tension provoquée par la présence des ions modifie l'amplitude du champ dipolaire de détection aux bornes du circuit oscillant et l'interaction avec les ions devient plus compliquée que celle décrite par l'expression (1).

- Le champ de charge d'espace ionique devient important à des pressions élevées, et s'oppose au confinement d'autres ions. La limite supérieure du nombre 
d'ions confinés étant proportionnelle à la masse de l'ion [5], cette limite est plus élevée pour les ions $\mathrm{N}_{2}^{+}$ que pour les ions $\mathrm{N}^{+}$.

- Les collisions des ions avec leur gaz parent jouent un rôle de plus en plus important lorsque la pression augmente. Le faisceau électronique, qui traverse la cage, crée un nombre d'ions proportionnel à la pression, mais le taux de pertes supplémentaires dues aux collisions, augmente rapidement. Ce phénomène expliquerait la décroissance des courbes au-delà de la saturation.
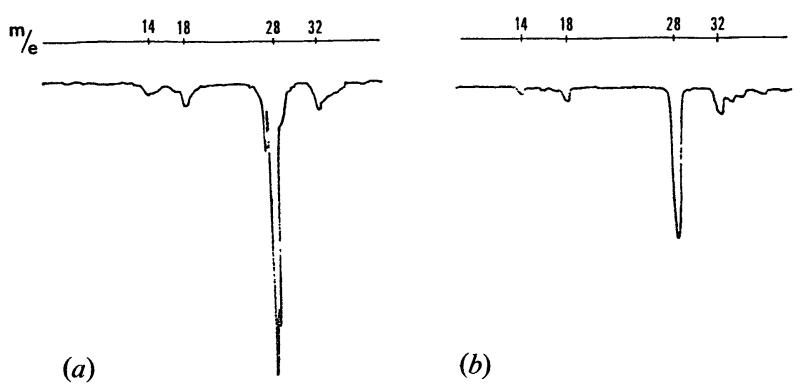

Fig. 10. - Spectres obtenus avec la cage (a) et le quadrupôle Riber $(b)$ après injection d'azote sous une pression partielle de $8 \times 10^{-9}$ torr. Vide résiduel : $5 \times 10^{-9}$ torr. Pour le premier spectre : sensibilité verticale de l'enregistreur : $50 \mathrm{mV} / \mathrm{cm}$. Pour le deuxième : sensibilité verticale de l'enregistreur : $5 \mathrm{mV} / \mathrm{cm}$.

[Spectra obtained with the cylindrical trap $(a)$ and the Riber quadrupole $(b)$ after injection of $8 \times 10^{-9}$ torr of nitrogen. Limit pressure : $5 \times 10^{-9}$ torr vertical sensibility of the $x-y$ recorder : for the first spectrum : $50 \mathrm{mV} / \mathrm{cm}$ for the second : $5 \mathrm{mV} / \mathrm{cm}$.]

Sur la figure 10 nous montrons deux spectres obtenus respectivement avec la cage et avec le quadrupôle Riber, après injection de $8 \times 10^{-9}$ torr d'azote. Pour le spectre obtenu avec la cage, la sensibilité verticale de l'enregistreur était $50 \mathrm{mV} / \mathrm{cm}$ et pour celui obtenu avec le quadrupôle $5 \mathrm{mV} / \mathrm{cm}$. Le rapport signal/bruit du pic de $m / e=28$ était 24 pour le quadrupôle et 28 pour la cage. Il faut tout de même noter que la sensibilité de la cage n'était pas meilleure que celle du quadrupôle en ce qui concerne les pics des ions dont le gaz était sous une très faible pression partielle à l'intérieur de la cage. Sur le spectre obtenu avec le quadrupôle on voit un plus grand nombre des pics que sur le spectre obtenu avec la cage.

La résolution, définie comme le rapport $m / \Delta m$ de la masse $m$ sur la largeur du pic à sa mi-hauteur, mesurée sur le pic 28 , était :

- pour la cage : résolution 29

- pour le quadrupôle Riber : résolution 36.

En général, la résolution que l'on obtient avec la cage est inférieure à celle du quadrupôle. La légère variation de la fréquence d'oscillation des ions du même rapport $m / e$ en fonction de leur point de création limite en effet la résolution en élargissant les pics [5]. Des ions de masse voisine peuvent être confondus avec l'ion observé. b) Les variations de hauteur des pics d'argon à une ou deux charges, $\mathrm{A}^{+}$et $\mathrm{A}^{++}$, ont été également tracées en fonction de la pression partielle d'argon (Fig. 11). La tension de détection utilisée était de $10 \mathrm{mV}$ entre les deux chapeaux.

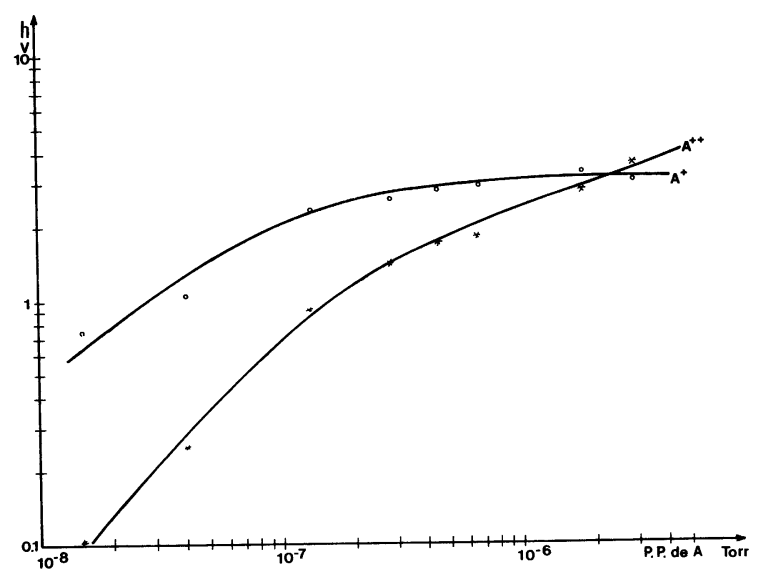

Fig. 11. - Variation de la hauteur des pics d' $A^{+}$et $A^{++}$en fonction de la pression partielle d'argon injecté extérieurement. Tension de détection : $V_{\mathrm{d}}=5 \mathrm{mV}$ sur chaque chapeau.

[Variation of the signal corresponding to $A^{+}$and $A^{++}$as a function of the partial pressure of argon. Detection voltage : $5 \mathrm{mV}$ on each end-cap.]

Les ions $\mathrm{A}^{++}$ayant une charge deux fois plus élevée que celle de $\mathrm{A}^{+}$, leur densité maximum permise [5], proportionnelle au rapport $m /(Z e)^{2}$, devrait être 4 fois plus faible. Mais le signal ionique observé à la résonance devrait être selon l'expression (1), deux fois plus élevé que le signal correspondant au même nombre d'ions $\mathrm{A}^{+}$. Au total, si la saturation était uniquement due à la charge d'espace ionique, on devrait s'attendre à un signal maximum deux fois plus faible avec $\mathrm{A}^{++}$ qu'avec $\mathrm{A}^{+}$. Or, d'après la figure 11 , l'évolution avec la pression du pic $\mathrm{A}^{++}$ne suit pas cette prévision. La hauteur du pic $\mathrm{A}^{++}$(qui se situe en $m / Z e=20$ sur l'échelle des masses) augmente et à partir de $2,4 \times 10^{-6}$ torr, il devient plus important que le signal de saturation du pic de l'ion $\mathrm{A}^{+}$, et la saturation du signal ionique des ions $\mathrm{A}^{++}$sera obtenue pour une valeur plus élevée de la pression que celle des ions $\mathrm{A}^{+}$.

Ce phénomène est difficile à expliquer parce qu'il faudrait tenir compte de plusieurs facteurs qui interviennent simultanément, comme l'amplitude de la tension de détection, l'éventuelle présence d'ions parasites contribuant à la charge d'espace de l'ion observé, le courant électronique d'ionisation qui dépend de la tension $\mathrm{HF}$ de confinement, servant à accélérer les électrons, et de la droite de balayage utilisée (parce que selon la droite choisie les ions $\mathrm{A}^{+}$ peuvent ou non être stables pendant l'observation des ions $\mathrm{A}^{++}$et dans ce cas des ions deux fois chargés peuvent être formés à partir des ions chargés une fois).

Nous avons constaté que tous ces facteurs jouent sur le rapport $\mathrm{A}^{+} / \mathrm{A}^{++}$et nous avons examiné plus 
en détail ce rapport en fonction de la tension de détection appliquée. En effet, en regardant la variation de la hauteur des pics $\mathrm{A}^{+}$et $\mathrm{A}^{++}$à pression fixe $\left(8 \times 10^{-6}\right.$ torr après injection d'argon, vide limite $2 \times 10^{-9}$ torr) en fonction de la tension de détection, nous avons constaté que si la tension de détection est très faible $\left(\leqslant\right.$ à $12 \mathrm{mV}$ ) le pic correspondant à l'ion $\mathrm{A}^{++}$est plus élevé que le pic correspondant à l'ion $\mathrm{A}^{+}$. A la limite, avec une tension de détection nulle, nous avons même pu obtenir un pic important d'ions $\mathrm{A}^{++}$de signe opposé à celui obtenu en présence de tension de détection, ce qui montre que l'échange d'énergie entre les ions et le circuit oscillant de détection peut, en absence de champ dipolaire, conduire à une absorption d'énergie par le circuit oscillant. Ce phénomène inverse, conduisant à un refroidissement des ions a déjà été décrit par H. G. Dehmelt et F. L. Walls [10] pour la trappe statique de Penning mais il n'avait jamais été observé expérimentalement avec la cage radiofréquence. Avec les ions $\mathrm{A}^{+}$le pic inverse, en absence de tension de détection, n'a pu être observé qu'avec un courant d'ionisation très élevé, mais dans tous les cas le pic de $\mathrm{A}^{++}$a toujours été beaucoup plus grand que le pic $\mathrm{A}^{+}$. Probablement, l'absence de champ dipolaire, qui a une influence destructive sur la stabilité des ions, conduit à un temps de confinement des ions $\mathrm{A}^{+}$plus élevé, et on peut supposer que le processus de production des ions $\mathrm{A}^{++}$à partir des ions $\mathrm{A}^{+}$par collisions successives avec les électrons du faisceau devient important.

En augmentant la tension de détection, le rapport des hauteurs de pics $\left(\mathrm{A}^{+} / \mathrm{A}^{++}\right)$dépasse l'unité et augmente rapidement (Fig. 12).

Contrairement à la cage, le quadrupôle Riber donne un rapport hauteur du pic $\mathrm{A}^{+} /$hauteur du pic $\mathrm{A}^{++}$ à peu près constant indépendamment de la pression du gaz neutre.

4. Conclusion. - Les résultats expérimentaux obtenus nous montrent que pour de faibles pressions partielles, une cage cylindrique, utilisée avec un système simple de détection par absorption résonnante d'énergie, peut offrir une bonne sensibilité lorsqu'on travaille au point optimum du diagramme de stabilité [11], d'où l'utilisation possible de la cage comme détec-

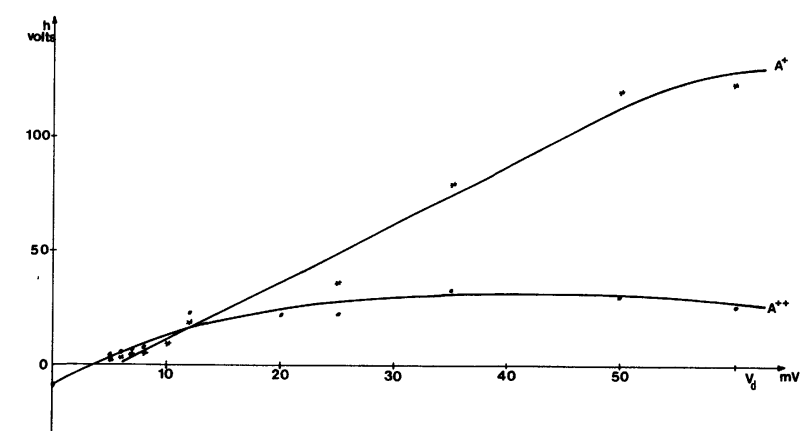

Fig. 12. - Variation de la hauteur des pics $\mathbf{A}^{+}$et $\mathbf{A}^{++}$en fonction de la tension de détection appliquée sur chaque chapeau.

[Variation of the ionic signal corresponding to $\mathrm{A}^{+}$and $\mathrm{A}^{++}$as a function of the detection voltage.]

teur de fuite, ou analyseur de gaz résiduels en ultravide.

A des pressions élevées, il faut utiliser une tension de détection plus élevée, pour pouvoir détecter la totalité du nombre d'ions piégés. Mais il faut noter que les champs de charge d'espace ionique et les collisions des ions avec les particules neutres deviennent importants à partir d'une pression de $10^{-7}$ torr et saturent le signal ionique observé. Afin de repousser cette saturation à des pressions plus élevées on pourrait déplacer la droite de balayage de façon qu'elle ne passe plus par le point optimum du diagramme de stabilité, mais par un point situé près de la limite supérieure ou inférieure de ce diagramme. De cette façon on se situerait dans une région du diagramme de stabilité où le rapport ions confinés/ions créés serait plus faible et la saturation due à la charge d'espace ionique serait ainsi atteinte pour des pressions partielles plus élevees. De plus le nombre d'espèces ioniques simultanément stables pendant l'observation d'un ion serait plus faible et on diminuerait ainsi l'influence d'une charge d'espace ionique parasite et les interactions entre ions (transfert de charge, etc.).

On note également l'importance d'un dégazage poussé de la cage pour éviter les ions parasites qui, s'ils sont stables pendant l'observation d'un ion donné, accroissent la charge d'espace dans le piège et diminuent donc la sensibilité.

\section{Bibliographie}

[1] Paul, W., Osberghaus, O., Fischer, E., Forschungsber. Wirtsch. Verkehrminist., Nordrheim-Westfalen, $\mathrm{n}^{\mathrm{o}} 415$ (1958).

[2] Benilan, M.-N., Audoin, C., Int. J. Mass Spectr. Ion Phys. 11 (1973) 421-432.

[3] Plumelle, F., Thèse de $3^{\text {e }}$ cycle, Université Paris-XI, Orsay (1979).

[4] Bonner, R. F., Fulford, J. E., MarCh, R. E., Int. J. Mass Spectrom. Ion Physics 24 (1977) 255-269.
[5] Galiouna Nassiopoulos, A., Moller, P. A., Septier, A., Première partie de cet article Revue Phys. Appl. 15 (1980)
1529.

[6] Major, F. G., Dehmelt, H. G., Phys. Rev. 170 (1968) 91.

[7] SchweBel, C. E., Thèse de $3^{\mathrm{e}}$ cycle, Orsay (1974).

[8] Manuel $\mathrm{n}^{\circ}$ M6008, Instrumentation ultravide « Riber ».

[9] ANDRÉ, J., Thèse d'Etat, Université de Marseille, Mai (1978).

[10] Dehmelt, H. G., Walls, F. L., Phys. Rev. Lett. 21 (1968) 127.

[11] Galiouna Nassiopoulos, A., Thèse de $3^{\mathrm{e}}$ cycle, Orsay (1980). 